\title{
Zentrifugen des Wissens Zur Enzyklopädik des Barockromans
}

\section{Journal Article}

\section{Author(s):}

Kilcher, Andreas (1)

Publication date:

2013-11

Permanent link:

https://doi.org/10.3929/ethz-b-000075506

Rights / license:

In Copyright - Non-Commercial Use Permitted

Originally published in:

Arcadia 48(2), https://doi.org/10.1515/arcadia-2013-0021 
Andreas B. Kilcher

\section{Zentrifugen des Wissens - Zur Enzyklopädik des Barockromans}

Abstract: Early modern literature has high epistemological claims. In particular, the novel as the most innovative genre of the 16th and 17th centuries was expected to negotiate and transmit knowledge about the world in an extensive way. This epistemological optimism must be understood against the background of contemporary encyclopaedic models, which offered new possibilities of reaching out for universal and total knowledge. Two variants of encyclopaedic writing are most efficient for the novel: the logic of Lullism and the miscellaneous knowledge production of Polyhistorism. Both techniques were used in baroque novels of the 17th century: Polyhistorism produced a centrifugal dispersion of knowledge throughout the texts, whereas Lullism aimed at recollecting and ordering it. This interplay is evidently present in Daniel Casper von Lohenstein's highly digressive 3,000 page novel „Arminius“ (1689/90), with its paratextual framework of prefaces, annotations, and indices. Moreover, the reception of „Arminius“ in 18th and 19th centuries is pertinent for the subsequent critique of encyclopaedic knowledge.

Keywords: encyclopaedia, Lullism, polyhistorism, universality, Daniel Casper von Lohenstein.

Prof. Dr. Andreas B. Kilcher: Professur für Literatur- und Kulturwissenschaft, Eidgenössische Technische Hochschule Zürich, Rämistraße 101, 8092 Zürich, Schweiz, E-Mail: akilcher@ethz.ch

Die Behauptung einer Wissensfunktion der Literatur mag für die ästhetische Moderne unter dem Vorzeichen einer Autonomieästhetik seit der Goethezeit höchst begründungsbedürftig sein. Erst recht scheinen ein universaler Wissensanspruch wie derjenige der Enzyklopädie sowie seine formalen Umsetzungen nachgerade den denkbar größten Gegensatz zu einer schönen Literatur zu bilden, die sich grundsätzlich über den ästhetischen Freiraum als der Sphäre des „Interesselosen“ und „Zweckfreien“ definiert, wie dies etwa Kant, Moritz und Schiller in ihren klassischen Ästhetiken um 1800 gedacht haben. Die Literatur der Frühen Neuzeit zwischen Humanismus und Barock dagegen verhält sich zum Wissen fundamental anders: In keiner Weise stößt sie sich an einem epistemologischen Anspruch, sei er noch so umfassend. Vielmehr geht die vormoderne Poetik 
grundsätzlich davon aus, dass die Literatur und ihre Gattungen, insbesondere aber der noch junge Roman, in hohem Maße dazu geeignet sind, Wissen - auch und gerade in enzyklopädischen Formen und Funktionen - zu verhandeln und $\mathrm{zu}$ vermitteln.

Nicht nur vor diesem poetikgeschichtlichen, sondern auch vor einem wissensgeschichtlichen Hintergrund ist die formale und funktionale Engführung von Literatur und Enzyklopädie in der Frühen Neuzeit zu verstehen. Auch im Kontext eines neuen Optimismus über die Möglichkeiten des Wissens, in der Ausdifferenzierung insbesondere von neuen enzyklopädischen Modellen, wurde der Literatur die Aufgabe der Konstitution einer „Einheit des Wissens“ zugesprochen (vgl. Kilcher 2003, Müller-Bochat 1966). Namentlich die Großform des Romans im Barock ist wesentlich vor diesem Hintergrund der Behauptung neuer Möglichkeiten einer Universalität und Totalität des Wissens in der Frühen Neuzeit zu verstehen: Der Barockroman setzte enzyklopädische Modelle auf vielfältige Weise epistemologisch um und entwickelte sie poetologisch weiter. Andererseits ging auch die Ausdifferenzierung enzyklopädischer Modelle im Zeitalter des Buchdrucks mit neuen medialen, poetologischen und ästhetischen Möglichkeiten einher. Auch dies begründet in der Frühen Neuzeit die weitreichende formale wie funktionale Engführung von Literatur und Wissen am Modell der Enzyklopädie.

Im Folgenden ist diese Verbindung in drei Schritten zu analysieren: erstens an der Ausdifferenzierung enzyklopädischer Modelle mit ihren Ordnungs- und Schreibweisen im Umfeld der Frühen Neuzeit, zweitens an deren Funktion für die Gattung des sogenannten polyhistorischen Romans als der privilegierten enzyklopädischen Form der Literatur im Barock, drittens schließlich an der Kontroverse über den polyhistorischen Roman, wie sie vom späten 17. bis ins 19. Jahrhundert geführt wurde.

\section{Modelle der Enzyklopädie in der Frühen Neuzeit}

Jene Einheit des Wissens, die seit dem 15. Jahrhundert mit dem Begriff der encyclopaedia bezeichnet wurde und seit Paul Scaligers Encyclopaedia, seu orbis disciplinarum (1559) auf Buchtiteln erschien, ließ sich in Humanismus und Barock in unterschiedlichen Modellen denken. Besonders drei lassen sich unterscheiden: die systematische Anordnung der einzelnen Wissenschaften, die logische Begründung des Wissens überhaupt, die historisch-philologische Ansammlung von Wissensgegenständen.

Diese grundlegend verschiedenen Modelle waren für die Entwicklung des Romans unterschiedlich prägend. Weniger relevant für den Roman war die seit der Antike dominante disziplinäre Form enzyklopädischer Wissensordnung: das 
System der artes liberales, der sieben Freien Künste. Zwar hatte dieses System, das spätestens mit Martianus Capellas De nuptiis Philologiae et Mercurii (6. Jh.) kanonisiert wurde, in der ersten frühneuzeitlichen Enzyklopädie eine herausragende neue Ausgestaltung erhalten, die im Zeitalter des Buchdrucks auch neue Möglichkeiten des Bildeinsatzes erprobte: Gregor Reischs Margarita philosophica (1503) (vgl. Siegel 2004). Einflussreich war diese Enzyklopädie aber auch damit, dass sie das klassische System der artes liberales, das die drei Buchstabenkünste (Grammatik, Rhetorik, Dialektik) und die vier Zahlenkünste (Arithmetik, Geometrie, Musik, Astronomie) umfasst, um die Wissenschaften der Natur (philosophia naturalis) und des Menschen (philosophia moralis) erweiterte. Damit war die Margarita philosophica prägend für die Herausbildung von Curricula an humanistischen Universitäten und Schulen, jedoch weniger für die Ausdifferenzierung enzyklopädischer Romanprosa.

Anders verhält es sich mit den beiden weiteren enzyklopädischen Ordnungsund Schreibmodellen der Frühen Neuzeit; diese waren für die Erzählform des Wissens und damit insbesondere für den Roman in hohem Maße prägend, auch wenn sie in der Logik der Wissensordnung geradezu konträr waren: auf der einen Seite der logisch-philosophische, auf der anderen Seite der material-philologische Typus. Sie bildeten damit zwei höchst unterschiedliche Narrative des Wissens aus: Das eine baut auf der rationalen Begründung des Vielen in einer Einheit des Wissens durch Relationierung von abstrakten Grundformen, das andere versammelt eine Vielzahl disparater Wissensgegenstände in dezidiert unsystematischen und kontingenten Formen. Die Rede ist vom Lullismus auf der einen und dem Polyhistorismus auf der anderen Seite, die beide die Literatur des Barock wesentlich mit leiteten.

\section{Lullismus}

Die logische Enzyklopädik baut auf der Relationierung einer begrenzten Anzahl abstrakter Elemente auf, um auf diese Weise eine potenzielle Totalität des Wissens zu konstituieren. Das Paradigma dieses topischen Universalsystems ist dasjenige, das der Mallorquiner Ramon Lull um 1300 als Ars in mehreren Fassungen entwickelt hatte und das sodann in der Frühen Neuzeit zu einem leitenden universalwissenschaftlichen Modell wurde. Lulls Wissenskunst basiert einerseits auf der Festlegung einer Anzahl von Elementen (Grundbegriffe, Substanzen etc.), andererseits auf der Art und Weise, wie diese Elemente miteinander in Relation gebracht werden. In der Hauptfassung der Ars, der Ars generalis ultima, sowie in deren Kurzfassung, der Ars brevis (beide 1308 beendet), sind die Elemente auf neun absolute Prinzipien (dignitates) reduziert, die sodann mit neun relativen 
Prinzipien (die in Teilen den aristotelischen Kategorien entsprechen) sowie mit neun Fragen, Subjekten, Tugenden und Lastern auf kombinatorische Art und Weise korreliert werden.

Diese kombinatorische Verknüpfung von Elementenreihen setzt Lull in einem „logischen Apparat“ um, wobei die Elemente zu heuristischen Zwecken mit den neun Buchstaben B bis K bezeichnet werden. Leitend ist dabei das Prinzip der Kombinatorik, das es erlaubt, aus der Verbindung von Elementen eine potenzielle Totalität - bzw. eine Totalität an Möglichkeiten - zu generieren. Lull systematisiert diese Möglichkeiten in vier Figuren, die den Kern des „logischen Apparats“ bilden. Die erste kombiniert zwei, die zweite drei Reihen zu je neun Elementen. „In den Prinzipien dieser Figur“, so Lull schon zu der ersten, „ist alles enthalten, was ist“ (Lullus 1999, 9). Lulls kombinatorische Apparaturen sollen dem eigenen Anspruch nach jedoch nicht etwa nur alles aktuelle Wissen versammeln, sondern vielmehr alles je mögliche Wissen konstruieren.

Nicht zuletzt diese Erweiterung der Enzyklopädik von einem faktischen und realen Wissen hin zu einem Möglichen des Wissens begründet die besondere Attraktivität von Lulls universaler Topik in der Frühen Neuzeit. Als Lullismus ließ sie sich in Universalsprachentheorien ebenso wie in mathematischen Zeichenmodellen ausdifferenzieren: Giordano Bruno erweiterte Lulls Topik zu einer esoterischen Mnemotechnik (vgl. Yates 1964), der barocke Universalgelehrte Athanasius Kircher rationalisierte die lullische Ars in seiner Ars magna sciendi sive combinatoria (1669) zu einer Enzyklopädik und Leibniz begründete mit ihr sein universalsprachliches Projekt einer Characteristica Generalis, die mit einem logischen Zeichensatz qua ars combinatoria eine Totalität aller möglichen Aussagen konstruieren sollte (vgl. Schmidt-Biggemann 1983; Rossi 1960, 1961 u. 1984). Diese Präsenz der lullischen Ars in der Frühen Neuzeit belegt u.a. auch ihre Aufnahme in Tomaso Garzonis populärer Enzyklopädie der praktischen Künste, der Piazza universale, Das ist: Allgemeiner Schawplatz / oder Marckt / vnd Zusammenkunfft aller Professionen / Künsten / Geschäfften / Händeln vnd Handtwercken / so in der gantzen Welt geübet werden (italienisch 1585, deutsch 1619). Unter dem Titel „Kunst Raymundi Lullii“ wird sie hier - wenn auch aus der Sicht der praktischen Künste nunmehr kritisch - als ein aktuell weit verbreitetes Verfahren vorgestellt, das verspricht, ,alle Künste auff einmal / oder ja in gar kurtzer Zeit zu fressen oder zu greiffen“ (Garzoni 1626, 141). Eben dieses Versprechen der Totalisierung bewirkte noch zwei Jahrhunderte später die Attraktivität des Lullismus für das kombinatorische Enzyklopädieprojekt von Novalis, das ebenfalls unter dem Schlagwort des Allgemeinen firmiert (Allgemeines Brouillon, 1798/99; vgl. Neubauer 1978; Moser 1989).

Bereits in der Frühen Neuzeit wurde die lullische Ars auch poetologisch fruchtbar gemacht. Tatsächlich ließ sie sich nicht nur als universalistisches 
Wissens- und Sprachmodell, sondern auch als poetologische Schreibtechnik verstehen. Aus der ars magna sciendi wurde in den barocken Poetiken der „Letterwechsel“ wie etwa bei Georg Philipp Harsdörffer (Harsdörffer 1968/1969: 183; vgl. auch Zeller 1974). Im Anschluss an Kircher setzte insbesondere Quirinus Kuhlmann die ars combinatoria als eine universale, zugleich epistemologische, ontologische und poetische Methode um. So verstand er in Geschicht-Herold (1673) die „Wechselkunst“ „nach der neuvermehrten Wundersart des weltberufenen Athanasius Kircher“ nicht nur als das universale Prinzip „aller Wissenschaften Wissenschaft“ sowie des „Naturwechsels“ (Kuhlmann 1673, Vorgespräche Nr. 7); mit Kirchers „Centralzergliederungskunst“ oder „Centralwissenschaft“ begründete er zugleich auch eine neue linguistische und poetische „Sprachkunst“: „Komm herbei! O Sprachkunst! mit deinem Buchstabenheere! Ist deine Aneinanderfügung nicht alleine ein eintziges Wechselrad?“ (Kuhlmann 1673, Vorgespräche Nr. 23; vgl. zur Korrespondenz mit Kircher: Kuhlmann 1674).

Poetisch umgesetzt hat dies Kuhlmann allerdings nicht in einem Roman, sondern in einem experimentellen Text, der eine Art Prosagedicht darstellt, zugleich aber potenziell ins Große angelegt ist: dem „Wechsel-kus“, einem der Himmlischen Libes-küsse (1671). Diesen spielerischen Text leitet er mit einer philosophischen Bemerkung ein, die die Kombinatorik erneut als Universalgesetz der Welt und des Lebens vorstellt, überschrieben mit „der Wechsel Menschlicher Sachen“: „Nihil est in rebus humanis Natura stabile, nihil aequabile, nihil sufficiens, nihil in eodem statu permanens; sed omnia quadam veluti rota circumvolentur [...]““, was er deutsch verknappt auf den Punkt bringt: „Alles wechselt“ (Kuhlmann 1671, 53f.). Zur poetischen Umsetzung dieses Prinzips liefert Kuhlmann nicht nur die Elemente - nämlich Wörter - eines „volständigen Wechselsatzes“, sondern rechnet auch deren „Versetzung“ aus. Allein die Kombination seiner Elemente mit Wiederholungen ergibt über 622 Millionen Möglichkeiten, „zu welcher [...] Verwechselung der allerfertiste Scheiber / der täglich über tausend Verwechselungszeilen abschribe / doch über ein gantzes Jahrhundert sattsam würde zu schaffen haben“ (Kuhlmann 1671, 56ff.). Als Beispiel errechnet er die Permutation von 50 Wörtern nach dieser Methode und kommt dabei auf eine astronomische Zahl mit 77 Ziffern, die er auf über zwei Seiten ausschreibt (Kuhlmann 1671, 56-59). Zur Rationalisierung der Kombination konstruierte Kuhlmann daher, wie schon Lull in der quarta figura, einen kombinatorischen Apparat, bestehend aus drehbaren Alphabet-Scheiben: „ich [...] erfand darüber ein Wechselrad / durch das mein Reim / der in einem Jahrhundert ni ausgewechselt/ innert etlicheren Tagen völlig ausgewechselt“ (Kuhlmann 1673, Vorgespräche Nr. 19). Poetisches Schreiben ist hier - denkbar weit entfernt von Mimesis zu einem sprachlogischen Verfahren geworden, das aus der mathematisierbaren Fülle, dem pleroma eines mundus combinatus schöpft. 


\section{Polyhistorismus}

Während sich die lullische Kombinatorik, wie das Beispiel zeigt, eher in experimentellen lyrischen Texten umsetzen ließ, baute die erzählende Großform des Romans im 17. Jahrhundert vornehmlich - wenn auch nicht ausschließlich - auf der zweiten, komplementären Form der Enzyklopädie auf: der historisch-philologischen, dem Typus des Polyhistorismus. Auch dieser Typus ist keine Erfindung des Barock. Er gründet auf der antiken Gattung der poikile historia, der Buntschriftstellerei, die in dezidiert systemlosen Textformen wie Miszellen und Florilegien dem universalwissenschaftlichen Anspruch der „Polymathia“ folgt. Es ist dies eine eklektische Form von Wissensakkumulation, bei der es gerade nicht um logisch-rationale Begründung geht, sondern um die Sammlung und Vermittlung von Historien- und Realienwissen. Dieses ist, gemäß Aristoteles' Begriff der cognitio singularium, ein materiales Wissen etwa in der Form der historia naturalis oder der historia civilis. Solche singulären Wissensgegenstände werden dabei in kontingenten Formen zusammengeführt, unter Titeln wie „Thesaurus“, „Theatrum“, „Speculum“, „Museum“, „Florilegium“ etc. Das antike Paradebeispiel dafür, Aulus Gellius’ Noctes Atticae (um 170 n.Chr.), umschreibt den Inhalt solcher Sammlung programmatisch als ,allerhand bunte und mannigfaltig untermischte Lehren“ (,varia et miscella et quasi confusa doctrina“), indem „nach (eignem) Gutdünken alles nur Mögliche (d.h. Gelesenes und Gehörtes) ohne Ordnung und Unterschied“ angehäuft und „zur Unterstützung des Gedächtnisses eine Art Wissensvorrath“ angelegt wird; „so musste natürlich auch bei den vorliegenden Aufsätzen eine Buntscheckigkeit der Notizen entstehen“ (Aulus Gellius 1875, 1f.).

Dieser enzyklopädische Typus war im Humanismus und insbesondere in seinen Spätformen im Barock als Vermittlung und Popularisierung eines überwiegend aus Büchern gewonnenen Wissens weit verbreitet (vgl. Schock 2012). Dabei wurde der barocke Polyhistorismus nicht nur in Sachbüchern gefasst, sondern auch in literarischen Texten, insbesondere in der neuen Form des Romans. Mehr noch: Die Grenzen zwischen sachlich-faktualer Wissensliteratur und erzählerisch-fiktiver polyhistorischer Romanliteratur waren in inhaltlicher wie formaler Hinsicht fließend. So konnte die polyhistorische Wissensliteratur erzählerische Elemente enthalten. Beispiele dafür sind mehrbändige kompilatorische Werke, etwa die Frauenzimmer Gesprächspiele von Georg Philipp Harsdörffer (8 Bde., 1644-57) oder Eberhard Werner Happels schon dem Titel nach programmatisch polyhistorische Gröste Denckwürdigkeiten dieser Welt: Oder so genannte Relationes curiosae: Worinn dargestellet, und Nach dem Probier-Stein der Vernunfft examiniret werden, Alle so Physicalische, al $\beta$ Mathematische, Historische und andere merckwürdige Seltzamkeiten (5 Bde., 1683-1691, vgl. Schock 2011), sowie 
sein Mundus Mirabilis Tripartitus, Oder Wunderbare Welt, in einer kurtzen Cosmographia fürgestellet...: Welcher handelt Von den Menschen auf der Welt, von ihren Dignitäten, Potentaten, Religionen, Estats-Maximen, Macht, Intraden, Kriegs-Art, Waffen, Policey, Regiments-Geschäfften, Wahl-Ceremonien, Krönungen, Kleidungen, Sitten, hohen Gerichts-Kammern,...: Ein Werck vor Junge und Alte, Hohe und Niedere, Gelährte und Ungelährte, höchst- nutz- und ergötzlich zu lesen (16871708).

Das epistemologische Programm dieses Polyhistorismus formulierte Georg Philipp Harsdörffer im „Vorbericht“ zum dritten Band der Mathematischen und Philosophische Erquickstunden (1653). Die Frage, „Ob besser sey/ von allem etwas/ oder eine Sache allein vollständig wissen verstehen?“ beantwortet er mit einer entschiedenen Apologie des „Vielwissens“: Während ein schwaches „Gehirn“ sich mit bloß einer Wissenschaft beschäftigen könne, vermöge das starke vieles zu wissen - in der Gewissheit, dass die Gegenstände des Wissens nicht isoliert, sondern untereinander vernetzt seien:

\begin{abstract}
„Des Menschen Verstand ist kein Gefäß / das sich also anfüllet / wie etwan ein Becher / darein eine Maas / und nicht mehr kann gegossen werden: Nein / er mag soviel nicht begreiffen, da er nicht noch viel einmehrers sollte fassen und lernen können / und solche unendliche Fähigkeit und Begierde zu lernen ist das Kennzeichen seiner überirdischen und fast Göttlichen Eigenschafft. Zudem hangen alle Künste aneinander / wie an einer Ketten / können und werden nicht wol gesondert, daß also derjenige / welcher nur eine studiren will / gleich ist einem Mann / der mit einem Kettenring die Wahrheit aus einem tieffen Brunnen schöpffen will [...]. Ja / man kan keine Sache grundrichtig erkundigen / man wisse dann von vielen andern zugleich; wie man keine absonderliche Landtafel verstehen kan / man habe ja die gantze Weltkugel zuvor gesehen / und derselben Zirckel unterscheiden lernen.“ (Harsdörffer 1653, 24f.)
\end{abstract}

Dieser quantitativen Behauptung des „Vielwissens“ folgt die qualitative Frage nach der Wissensordnung: „ob man nicht alle Wissenschaften Lehrartig in eine Verfassung bringen könne?“ Während die quantitative Frage des Vielwissens für den Polyhistorismus zu bejahen ist, zweifelt Harsdörffer konsequenterweise an den Möglichkeiten einer systematischen Wissensordnung. Der Polyhistorismus geht einher mit einer Skepsis gegenüber dem Ordnungsbegriff.

„Unsre Erkanntnis wie man keine machet keine solche Schlussrede / daß man aus dem vergehenden das folgende unfehlbar solte begreiffen können / und ist eine andre Sache / alle Wissenschafften in richtiger Ordnung Lehrartig begreffen / eine andre / aus allen Wissenschafften eine einige machen.“ (Harsdörffer 1653, 27)

Dieses Modell eines a-systematischen polyhistorischen Vielwissens ließ sich nicht nur für die Wissenschaften im Allgemeinen, sondern insbesondere auch für die humanistische Philologie behaupten, die sich an der Literatur bildete. Diese Philo- 
logie, die sich auf den Universalgelehrten und Bibliothekar von Alexandria Eratosthenes von Kyrene zurückschreibt, ist ihrem Anspruch nach polyhistorisch: Ihr Wissen ist wesentlich aus Büchern gewonnen und umfasst ein historisch-geographisch-antiquarisch-naturkundliches Feld, das nicht systematisch geordnet, sondern miszellaneisch gesammelt wird. Im humanistischen Wissen wurde die Philologie damit zum Paradebeispiel eines Polyhistorismus, der - mit Homers Epen vor Augen - wesentlich aus der Literatur schöpft und sich in ihr entfaltet. Die humanistische Philologie differenzierte genauer drei Wissensformen aus: eine sprachlich-formale (Grammatik), eine philosophisch-kritische (Kritik) und eine historisch-antiquarische (Polymathie). Mit Blick darauf versteht der französische Philologe, Humanist und Bibliothekar Guillaume Budé in De philologia 1532 diese Wissenschaft als eine umfassende, indem sie alle anderen integrieren kann: „Die Philologie umfaßt alle anderen Disziplinen und hält in ihrem Kreis zusammen, was sie als einzelne in ihren Grenzen gleichsam mit dem Rechte des Architekten umschreibt“ (Budé 1557, 48). In seiner 1576 gehaltenen akademischen Rede De barbarie imminente (1578) - um ein zweites Beispiel zu geben - behauptet Caspar Hofmann, Medizinprofessor in Frankfurt an der Oder, ebenfalls eindringlich dieses polyhistorische Programm der humanistischen Philologie:

\footnotetext{
Philologie ist also jene Erforschung und Betrachtung der Wörter sowie denk- und wissenswürdiger Sachen bei den Autoren sowie besonders die Kenntnis des Altertums. Außerdem die Erläuterung von Sentenzen, die Kommentierung von Gedichten, Apophtegmata, Sprichwörtern, Fabeln, Historien, Exempeln, die Befassung mit der Chronologie, der Geschichte, mit berühmten Kriegen, bedeutenden Menschen, der Natur der Lebewesen, mit dem Münzwesen und dergleichen mehr, was nicht jedem sofort auf der Hand liegt. Dann auch die Beschreibung von Sachen, Flüssen, Bergen, Landschaften, Städten, und was dort in jeder rühmlichen Kunde überantwortet ist. Darauf beziehen sich auch die Einrichtungen der Sitten, die Kultur der Völker und Stämme, die Gewohnheiten des Altertums, die Behörden, Gerichtsformen, religiösen Rituale, die Dinge des Landbaus, der Stadt, des Hauses, des öffentlichen Lebens: dies alles aus den guten Autoren zu vermerken ist die Aufgabe des Philologen. (Hofmann 212)
}

Ihr polyhistorisches Programm sahen die humanistischen Philologen zunächst am Epos, beispielhaft an Homers Epen, erfüllt. Im Barock aber übernahm der Roman dieses Erbe: Er wurde zur privilegierten Gattung des Polyhistorismus.

\section{Der enzyklopädische Roman}

Der Roman des Barock ist nicht nur vage in diesem Umfeld eines Optimismus des Wissens in der Frühen Neuzeit angesiedelt. Er greift sehr präzise den universalwissenschaftlichen Anspruch des Lullismus und des Polyhistorismus auf. Primär 
relevant für den Roman ist zunächst vor allem letzterer, mithin der Wissensanspruch, den die polyhistorische Philologie vorzüglich in der Literatur realisiert sah. Das Programm eines in vielfältigen Exempeln, Exkursen und Zitaten gesammelten, nicht-systematischen Wissens ließ sich im 17. Jahrhundert besonders gut im Narrativ des Romans umsetzen. Der Roman erweist sich nachgerade als die Idealform eines Wissens, das in Elemente zerstreut ist und sich - in der Bruchgestalt von Exempeln und Exkursen - in einen noch so verwickelten Faden des Erzählens einflechten ließ.

Der polyhistorische Roman des Barock entwickelt dabei zwei komplementäre epistemologische Strategien: auf der einen Seite eine primäre und dominante zentrifugale Logik, indem ein vielfältiges, tendenziell universales Wissen im Text des Romans systemlos angesiedelt, ja nachgerade zerstreut wird; auf der anderen Seite eine sekundäre zentripetale Logik, die das über den Roman zerstreute Wissen förmlich einsammelt, den Wissenstext des Romans so navigierbar macht und zu diesem Zweck eigene enzyklopädische Ordnungssysteme einsetzt. Mit der ersten Logik folgt der Roman dem Muster der Polymathie; er erscheint gewissermaßen als eine Zentrifuge des Wissens. Mit der zweiten, zentripetalen Logik greift der Roman unterschiedliche enzyklopädische Formen und Schreibweisen der (Re)-Organisation auf, darunter insbesondere das alphabetische Register, aber auch die Verfahren der lullischen Topik (vgl. Link-Heer 1998).

Die Basis zur Ausdifferenzierung polyhistorischer und enzyklopädischer Formen und Funktionen des Romans definierte der Bischof von Avranches Pierre Daniel Huet im Traité de l'origine des romans (1670), einem der ersten romantheoretischen Entwürfe überhaupt, im Bildungsauftrag des Romans (vgl. Vosskamp 1973). Für den Roman gelte der Vorrang eines pädagogischen prodesse vor dem delectare: „Den vornehmsten Zweck der Romanen, oder welches zum wenigsten derselbe sein sollte, [...] ist die Unterrichtung in einigen Dingen oder Wissenschafften“ (Huet 1970, 32). Mit seinen literarischen Mitteln erhält der Roman jedoch zugleich einen entschiedenen Vorzug gegenüber der rein sachlichen Wissensliteratur. Seine erzählerischen und sprachlichen Mittel erfüllen die didaktische Funktion deshalb angemessener, weil „des Menschen gemüth von Natur eine feindin ist der Unterrichtungen [...], also muß man ihn locken, und betriegen durch die vergnügung, die Strengigkeit der unterweisung versüssen“ (ebd.). Das pädagogisch-didaktische Kalkül der „Unterrichtung in Dingen oder Wissenschafften" auf dem Weg des Romans liegt also darin, mit den erzählerischen Mitteln - dem Fingieren, dem Exemplifizieren, der gleichnishaften Übertragung etc. - neue, enzyklopädische Möglichkeiten der Bildung freizusetzen.

Dieser allgemeine romantheoretische Anspruch ist im Folgenden an einem Beispiel genauer zu erörtern. Es liegt nahe, dies an einem Beispiel des höfischen Romans, des GeschichtsGedichts, zu tun, stand hier doch der Bildungsauftrag im 
Zentrum. Eine umfangreichere Untersuchung müsste auch den „niederen“ Schelmenroman mit einbeziehen, der die enzyklopädische Bildungsfunktion aufgreift, zugleich aber karnevalisiert. An einem Beispiel wie Grimmelshausens Simplicissimus-Roman (1668) ließe sich etwa zeigen, wie das universale Wissen hier satirisch unter dem Vorzeichen des Nichtwissens erscheint, so im Bildungsprogramm des Simplicissimus am Eingang des Romans:

\begin{abstract}
in meinem zehen-jährigen Alter hatte ich schon die principia in obgemeldten meines Knans Adelichen Exercitien begriffen / aber der Studien halber konte ich neben dem berühmten Amplistidi hin passiren / von welchem Suidas meldet / daß er nicht über fünffe zehlen konte; dann mein Knan hatte vielleicht einen viel zu hohen Geist / und folgete dahero dem gewöhnlichen Gebrauch jetziger Zeit / in welcher viel vornehme Leut mit studiren / oder wie sie es nennen / mit Schulpossen sich nicht viel bekümmern / weil sie ihre Leut haben / der Plackscheisserei abzuwarten: Sonst war ich ein trefflicher Musicus auff der Sackpfeiffen / mit deren ich schöne Jalemj-Gesäng machen konnte: Aber die Theologiam anbelangend / laß ich mich nicht bereden / daß einer meines Alters damals in der gantzen Christenwelt gewest seye / der mir darinn hätte gleichen mögen / dann ich kennete weder GOtt noch Menschen / weder Himmel noch Höll / weder Engel noch Teuffel / und wuste weder Gutes noch Böses zu unterscheiden: Dahero ohnschwer zu gedenken / daß ich vermittelst solcher Theologiae, wie unsere erste Eltern im Paradis gelebt / die in ihrer Unschuld von Kranckheit / Tod und Sterben / weniger von der Auferstehung nichts gewust / O edels Leben! (du mögst wol Eselsleben sagen) in welchem man sich auch nichts umb die Medicin bekümmert. Eben auff diesen Schlag kan man meine Erfahrenheit in dem Studio legum und allen andern Künsten und Wissenschaften / so viel in der Welt seyn / auch verstehen; Ja ich war so perfect und vollkommen in der Unwissenheit / daß mir unmüglich war zu wissen / daß ich so gar nichts wuste. (Grimmelshausen 11f.)
\end{abstract}

Was der Schelmenroman nach dem Modell der menippeischen Satire sowie auch vor dem Hintergrund der Idee der docta ignoranita (vgl. Nikolaus von Kues 1977) karnevalesk als „enzyklopädisches Nichtwissen“ behauptet, ist im höfischen Staatsroman mit dem ernsthaften Anspruch auf universalhistorische Bildung vorgetragen. Besonders deutlich zeigt dies das Beispiel von Daniel Casper von Lohensteins Großmüthiger Feldherr Arminius oder Herman / Als ein tapfferer Beschirmer der deutschen Freyheit / Nebst seiner Durchlauchtigen Thüßnelda in einer sinnreichen Staats-Liebes- und Helden-Geschichte [...] vorgestellt. Der Roman des Anwalts, Diplomaten und Verfassers von zahlreichen Tragödien Lohenstein erschien 1689/90 - postum und unvollendet - in zwei Bänden mit je neun Büchern auf rund 3100 Seiten in Bearbeitung durch Lohensteins Bruder sowie durch den Leipziger Pastor und Theologen Christian Wagner. Er kann als eines der wichtigsten Beispiele des barocken polyhistorischen Romans gelten - neben den Romanen des Herzogs Anton Ulrich von Braunschweig-Wolfenbüttel Die Durchleuchtige Syrerinn Aramena (5 Teile, 1669-1673) und Octavia. Römische Geschichte (6 Teile, 1685-1707) (vgl. Heselhaus 1983, Kraft 2004). Lohensteins 
Arminius, auch ein patriotischer Schlüsselroman über die gesellschaftlich-politische Situation Deutschlands nach dem Dreißigjährigen Krieg, teilt mit Anton Ulrichs Romanen die Form des Polyhistorismus. Das Beispiel des Arminius ist dabei von besonderem Interesse, weil die zeitgenössische Debatte über die Wissensfunktion des Romans in erster Linie an ihm geführt wurde.

Tatsächlich setzte dieser Roman Huets Wissensforderung an den Roman in einer enzyklopädischen Steigerung um. Die Übereinstimmung mit jener Forderung zeigt schon der „Vorbericht an den Leser“ des Arminius, verfasst wahrscheinlich von dem Hoffmannswaldau-Herausgeber Benjamin Neukirch (Martino 1978, 212), indem er den Wissenserwerb zum eigentlichen Zweck des Romans erklärt und dabei den Roman als polyhistorisches Zirkulationsmedium versteht, das sich dadurch auszeichnet, dass es ein umfassendes geschichtliches Wissen mit literarischer Narration verbindet. So weiß er vom „Urheber“, dass dieser „viel des jenigen / was Er nicht bey den Geschichtschreibern gefunden / theils aus seinen alten Müntzen / theils aus den Uberschrifften und Gedächtnüs-Maalen / die er ihm insonderheit hierinnen überaus wol zu Nutz zu machen gewust / zusammen gesucht [...] welches der in den Alterthümern und Geschichten bewanderte Leser leicht mercken / die Räthsel auflösen / und die rechten Trauben von den gemahlten zu unterscheiden wissen wird“ (Vorbericht an den Leser, n.p.). Dieser „Urheber“ ist vornehmlich ein Kompilator, der Bücher aus Büchern schreibt und auf diese Weise seine Wissensgegenstände nicht entdeckt oder erfindet, sondern auffindet, einsammelt, erzählerisch vermittelt, kurz: narrativ in Zirkulation bringt:

gute Bücher [waren] seine unzertrennliche Gefärthen [...]. Massen sein Kopff ein rechtes Behältnüs der Wissenschafften zu seyn schien / darinnen er die allerwichtigsten BeweißGründe gesammelt hatte; und zu aller Zeit so wol aus dem Munde / als der Feder von sich geben / und gleichsam wie eine Schale den Balsam der Gelehrigkeit nur immer reichlich ausgiessen konte. [...] Was kan aber den Menschen eher tugendhafft machen / als gute Künste und Wissenschafften erlernen? (Vorbericht an den Leser, n.p.)

Während Lohenstein und seine Mitstreiter an dem monumentalen Romanprojekt Huets Vorgaben in der Didaktik und Enzyklopädik folgten, mehr noch: diese überboten, widersprachen sie ihm jedoch in der formalen und poetologischen Umsetzung des polyhistorischen Schreibens. Der Arminius-Roman verarbeitet sein Wissen gerade nicht in Form organischer „Haupthandlungen“, wie es Huet forderte, sondern gemäß dem Prinzip des Exkurses - seit Quintilian als digressio bekannt -, das er in einem Geflecht von „Nebenhandlungen“ umsetzt (vgl. Härter 2000). Was Lohensteins Roman zur Verdichtung der Daten einer „gantzen Bibliothec" macht, sind seine zahlreichen politisch-moralischen, naturwissenschaftlichen, geographischen, ethnologischen und historischen Exkurse (vgl. Kafitz 
1970, Szarota 1970). Diese Einschübe und Parallelerzählungen siedeln sich entlang einer weitläufigen „Staats- Liebes- und Helden-Geschichte“ um den Cheruskerfürsten Hermann an, deren Plot durch die permanenten Unterbrechungen immer neu verzögert wird und auf diese Weise schwer verfolgt werden kann. Auf der narrativen Ebene des Romans ist damit die verschachtelte Anlagerung von Exkursen die dominante Enzyklopädisierungsform. Auf diese Weise wird ein vielfältiges historisches, geographisches, ethnographisches, mythologisches, kunstgeschichtliches Wissen, das meist lateinischen Quellen entnommen ist, in die Haupthandlungen eingestreut. Der barocke Staatsroman integriert damit das Verfahren der poikile historia in die Literatur und wird so zu einem ,litteraten', einem hochgradig gelehrten und mit Wissen angereicherten Text: der Roman als Zentrifuge des Wissens.

Doch das über die Romanerzählung zentrifugal verstreute Wissen ist notwendig schwer nutzbar. Lohensteins Kritiker warfen ihm vor, dass der exzessive Einsatz der Digression sowohl die Romanerzählung lahmlege als auch das Gedächtnis überfordere und damit Wissen überhaupt verunmögliche (dazu ausführlicher der Schlussabschnitt dieses Aufsatzes). Im Bewusstsein des Problems hatten aber bereits die Herausgeber von Lohensteins Großroman tatsächlich eine Reihe von signifikanten schreibtechnischen Instrumenten entwickelt, die die zerstreuten Wissensdaten organisieren und den labyrinthischen, digressiv verschachtelten Romantext navigierbar machen sollten. Im Vordergrund stehen vor allem zwei gelehrte Schreibtechniken: Anmerkungen und Register. Im Hintergrund wird zudem auch die lullische Topik eingesetzt.

Hergestellt hat diese sekundären Instrumente zunächst Christian Wagner. Auf den zweiten Band des Romans ließ er die Anmerckungen über Herrn Daniel Caspers von Lohenstein Arminius nebst beygefügtem Register derer in selbigem Werck befindlichen Merckwürdigen Nahmen und Sachen (1690) folgen. Das Bedürfnis dieser enzyklopädischen Aufschreibesysteme erklärt Wagner präzise aus der Tatsache, dass Lohensteins Roman als sekundäre Form eines Wissensspeichers erscheint: „Gewiß ists / daß gleich wie der grundgelehrte Lohenstein eine lebendige Bibliothec gewesen / also dieses Buch ein rechter Kern und Auszug seiner gantzen leblosen Bibliothec mit allem Rechte heissen kan“ (Wagner 1690, $7 \mathrm{f}$.). Die 50 doppelspaltig bedruckten Seiten mit Anmerkungen verzeichnen intratextuelle, vor allem aber intertextuelle Bezüge des Romans. Sie lassen hinter dem Romantext palimpsestartig jene universalhistorische „Bibliothek“ aufscheinen, aus der der Roman schöpft, indem sie auf eine große Breite an Quellen zwischen historischer, naturwissenschaftlicher, theologischer und mythologischer Literatur rekurrieren. Die Anmerkungen legen die Anker zur Herkunft der vielfältigsten Wissensbausteine und machen den fiktiven Romantext so auch als einen faktualen Sachtext lesbar. Doch mit ihren verzweigten Exkursen tritt diese „Bibliothek“ 
in Romanform dem Leser als eine amorphe Datenmenge entgegen, ein Ozean an Information, der zu aktuellem Wissen erst dann verhelfen kann, wenn er navigierbar und damit nutzbar gemacht werden kann. Dies zu leisten ist in einer realen Bibliothek die Aufgabe des Katalogs. Im Roman wird diese Aufgabe von den Registern übernommen. Wagner hat diese daher ausdrücklich als Lösung des Problems der Datenmenge vorgestellt:

Dan zweiffelt nicht / es werde das gegenwärtige Leben des grossen Arminius / als des seeligen Herrns von Lohenstein vollkommenstes Meisterstück / bey gelehrter Welt in der sonderbaren Hochachtung jederzeit bleiben / die es lange zuvor nach seinem Verdienst erlangt hat / ehe es noch durch den Druck ans öffentliche Tages-Liecht gekommen ist. Jedennoch dörffte manchem vielleicht beschwerlich seyn / daß die Einführung unterschiedener seltzam-benennter oder ungenennter Personen dieses Werck hier und dar dunckel gemacht / und wäre demnach kein Wunder / wenn selbiger der Lust und Nutzens / so er aus Lesung der deutlichen Oerter dieses sonst so hochverlangten Buches schöpffen könte / lieber entbehren /als zugleich über der Auslegung der verdeckten Geschichte sich den Kopff zerbrechen wolte (Wagner 1690, 3).

Lohensteins Bearbeiter waren nicht die Erfinder der Einrichtung des Romanregisters. Als enzyklopädisches Schreibinstrument war es systemisch für den barocken Staatsroman. So enthält auch Philipp von Zesens Simson (1679) einen Blatweiser der merkwürdigsten Dinge. Doch ist das Register des Arminius qualitativ wie quantitativ weitreichender: Mit 158 Spalten, in der zweiten Auflage von 1731 auf 174 Spalten erweitert, gehört es zu den umfangreichsten seiner Art (vgl. Welzig 1976). Geboten werden unterschiedliche Register, in der Hauptsache aber das alphabetische Verzeichnüß der fürnehmsten in dem Arminius und der Thußnelda befindlichen Sachen und Personen - von „Abdankung vom Regiment“ und „Aberglaube treibt in die Flucht“ bis hin zu „Zweykampff der Tußnelda“ und „Zwistigkeiten der Druden“. Das Register hat genau genommen eine doppelte, zugleich narrative und epistemologische Funktion: Es verschlagwortet die durch die Exkurse fragmentierte und pluralisierte Romanhandlung und macht das im Romantext zerstreute Wissen zugänglich.

Über dieses Register hinaus bietet der Anmerkungs- und Registerband noch weitere Navigationshilfen des polyhistorischen Romans, darunter insbesondere eine Reihe von Tabellen mit Geschlecht-Registern, die die komplexen Verwandtschaftsverhältnisse der historisch-literarischen Figuren tabellarisch aufzeigen, u.a. zu Augustus und zu Hermann. Neben der asystematischen Pragmatik des Lexikons steht damit die genealogische Systematisierungsform der Tabelle. Darüber hinaus enthält der Anmerkungsband ein weiteres alphabetisches Verzeichnis: das Register einiger zur Zeit Arminius bekanter Länder/ Völcker/ Städte, Berge/ Wälder/ Flüsse. Dieses Register dient hauptsächlich dazu, die im Roman verschlüsselten Namen zu dekodieren, denn: „dero Nahmen sind öffters entweder 
mit Fleiss verschwiegen / oder verdrehet / oder zwar wahrhafftig / aber doch dem zugelegt / dem sie nicht gehören“ (Wagner 1690, 16). So klärt dieses Verzeichnis etwa darüber auf, dass mit der „Ubier-Stadt“ Köln gemeint ist.

Diese sekundären Ordnungssysteme des Wissens konstruieren hinter dem aus- und abschweifenden Romantext einen zweiten verdeckten Wissenstext. Während das Narrativ des Romans das Wissen zersplittert, sammeln die Anmerkungen, Register und Tabellen eben diese Splitter ein. Dabei eliminieren sie das epische Moment. Indem sie auf den Wissensgehalt des Romans zugreifen, machen sie aus dem abschweifenden, digressiven, erzählenden, fiktiven einen geordneten, pragmatischen, faktographischen Text. Dabei leisten die Register nicht nur die Rückbindung an ein romanexternes Wissen. Sie dokumentieren auch die Heraufkunft einer noch jungen enzyklopädischen Form, deren große Karriere sich im 18. und 19. Jahrhundert entfalten wird: das Lexikon. Ein beiläufiger Hinweis Wagners zum Register der Länder/Völcker/Städte, Berge/ Wälder/ Flüsse zeigt diesen Zusammenhang: „Will inzwischen iemand noch eigentlicher davon berichtet seyn/ der bediene sich hierzu des [...] Lexici, des gelehrten Baselschen Professors Johann Jacob Hoffmanns“ (Wagner 1690, 16). Gemeint ist das Lexicon universale (1677) des Gräzisten Hof(f)man, eine der ersten alphabetischen Enzyklopädien überhaupt. Die beiden Bände von Hofmanns Universallexikon registrieren ziemlich genau jene Daten, die Lohenstein auf dreitausend Seiten zweier Romanbände verstreut und Wagner im Romanregister alphabetisch versammelt: Daten historischer, geographischer, genealogischer, poetischer, philologischer und mythologischer Art. Allein der Titel von Hofmanns Lexikon zeigt dies: Lexicon universale historico-geographico-chronologico-poetico-philologicum/ continens/ historiam omnis aevi, geographiam omnium Locorum, Genealogiam principum Familiarum, addita ubique Chronologia tum veteri tum recentiore, Mythologiam insuper omnium Fabularum, Discussionem Philologicam illustrium circa haec occurrentium Difficultatum. Die großen Implikationen der Alphabetisierung des Wissens im Lexikon, wie sie inmitten der Aufklärung etwa durch Diderot und D'Alembert behauptet wurden - die Säkularisierung und Pragmatisierung des Wissens -, stehen hier noch nicht auf dem Plan (vgl. Kilcher 2007). Dennoch wird deutlich, dass die Attraktivität des Registers für den Roman in eben diese Richtung eines Paradigmenwandels hin zur pragmatischen und kontingenten Ordnung des Wissens zielt.

Genau in die Gegenrichtung scheint schließlich eine letzte enzyklopädische Form dieses Romans zu weisen: die lullische Topik. Es mag überraschen, dass der Arminius auch diese vormoderne logische Apparatur des Wissens integriert. Tatsächlich wird jedoch bei genauerem Hinsehen erkennbar, dass die Wissensdaten in diesem Großroman aus zwei mal neun Kapiteln auch nach der topischen Logik des Lullismus angeordnet sind. Allerdings sind die Elemente dieser Topik nicht 
mehr dieselben: nicht die absoluten göttlichen Prinzipien etc., sondern die historisch-geographischen Gegebenheiten. Aus der Beantwortung der rhetorischen Fragen nach Person, Ort und Zeit ergeben sich, wie Thomas Borgstedt überzeugend gezeigt hat, „für jedes Buch eine jeweils eigene Kombination von Personal, Geographie und Historie. Die Gesamtanlage zielt auf Vollständigkeit. Das Romanpersonal wird (nahezu) vollständig exponiert, die Geographie deckt sämtliche Erdteile ab, und die Historie erzählt die gesamte bekannte Geschichte unter Bezug auf die Deutschen“ (Borgstedt 1992, 285). Lohensteins literarische Enzyklopädik folgt damit zugleich hintergründig einem dergestalt historisierten und säkularisierten Lullismus. Während Kircher und Leibniz von Lulls Ars die rationale Konstruktion einer absolute Sprache und eines vollkommenen Wissens erwarteten, verknüpft Lohensteins Roman geschichtliche, geographische und politische Daten zu einem polyhistorischen Gewebe.

\section{Kontroverse über den polyhistorischen Roman}

Das Umfeld der Edition macht deutlich, dass der Arminius-Roman schon bei seinem Erscheinen wesentlich unter dem Aspekt der Speicherung, Verwaltung und Vermittlung von Wissen wahrgenommen wurde. Doch gerade diese Wissensfunktion war in der Rezeption des Romans im 18. und 19. Jahrhundert höchst umstritten. Die damit verbundene Kontroverse dokumentiert letztlich auch einen Umbruch im Literaturbegriff um 1700 überhaupt: Auf der einen Seite steht die Behauptung eines späthumanistischen Literaturkonzeptes, das dem polyhistorischen Anspruch und seinen enzyklopädischen Schreibweisen folgt, auf der anderen Seite die Kritik des barocken Polyhistorismus und seiner enzyklopädischen Schreibweisen in der Aufklärung.

Die erste Position vertrat Christian Thomasius in seiner Rezension des Arminius von 1688 und 1689, wenn er den Roman zunächst als hervorragendes Organon der Bildung verteidigte: In seinem enzyklopädischen Narrativ sah er ein neues Lernen an „Exempeln“ umgesetzt, dessen Vorzug er darin erkannte, Wissen nicht nur „angenehmer“, sondern auch besser memorierbar zu machen. Mit Blick auf den Roman hebt Thomasius diesen didaktisch-mnemotechnischen Vorzug insbesondere für literarische Werke von universalhistorisch-enzyklopädischem Format hervor:

des Menschen Verstand auf dieser Welt [ist] so geartet [...], daß er alle Künste und Wissenschafften mehr durch Exempel als Regeln erlernet, und sich imprimiret [...]“" (Thomasius 1970, 44f.); „[...] der Herr von Lohenstein setzt uns in seinem Buch lauter gelehrte/ scharffsinnige und tugendhaffte Sachen vor/ und überziehet dieselbigen nur mit etwas von einer angenehmen invention. Es giebt der lehrbegierigen Jugend das thee der Weißheit zu trin- 
cken/ [...] so thut er ein wenig Zucker allerhand Historischer und Politischer inventionen hinein/ ümb ihnen den appetit zu erwecken. Ich kan wohl sagen/ daß ich kein Buch in der Welt weiß/ darinnen ich soviel Gelahrheit beysammen angetroffen [...]. (Texte zur Romantheorie I, 275)

Eine Apologie des Romans als ,Schatzkammer‘ des Wissens leisteten im Folgenden vor allem Christian Schröter und Johann Christoph Männling, ein Bewunderer und Schüler Lohensteins. In eigenen Kollektaneen zum Arminius-Roman erschließen sie, wie die Romanregister, den digressiven Polyhistorismus mit lexikographischer Technik: Sie transponieren das im Roman verstreute Vielwissen in die arbiträre Ordnung des Wörterbuchs. Männling, der später auch ein Poetisches Lexicon (1719) vorlegen sollte, organisierte die überbordende Wissensmenge des Arminius - in Überbietung der Romanregister - in eigenen enzyklopädischen Registraturen von res und verba. 1708 erschien der Arminius enucleatus. Das ist: Des unvergleichlichen Daniel Caspari von Lohenstein/ Herrliche realia, Köstliche Similia, Vortreffliche Historien/ Merkwürdige Sententien, und sonderbare Reden. Als Köstliche Perlen und Edelgesteine aus dessen deutschen Tractati oder Arminii. 1710 folgte ein zweites Lexikon, das das Vorhaben der Wort- und Sach-Thesaurierung auf alle Schriften Lohensteins ausdehnte, der Lohensteinius sententiosus, Das ist: Des vortrefflichen Daniel Caspari von Lohenstein/Sonderbahre Geschichte/ curieuse Sachen/ Sinn-reiche Reden/ durchdringliche Worte/ accurate Sententien, Hauptkluge Staats- und Lebens-Regeln/ und andere befindliche Merckwürdigkeiten/ Aus dessen sowohl Poetischen Schrifften und Tragoedien, als auch Lob-Reden/ und andern ihm zustehenden gelehrten Büchern/ Wie aus einem verborgenen Schatze zusammen colligiret/ Und der gelehrten Welt zur Verfügung/ der Jugend zum nützlichen Gebrauch Nebst einem vollkommenen Register ans Tage-Licht gestellet (vgl. auch Rieger 1997). Wie Wagner den Arminius als „einen rechten Kern und Auszug einer gantzen leblosen Bibliothec“ verstand, verspricht Männling im Arminius enuncleatus ,,als in einer Nuß den vollen Kern des grossen Arminii [...] beysammen ant[zu]reffen“ (Männling 1708, Vorrede). Männlings Enzyklopädisierungskalkül erweitert damit die Praxis sekundärer Extraktion, Thesaurierung und Registratur eines Wissens, das digressiv auf dreitausend Romanseiten verstreut wurde, vollends expandiert jedoch eine ganze Bibliothek füllte.

Diesen Apologien des Aminius folgte noch die zweite Ausgabe des Romans von 1731, die der Professor der Rechte in Leipzig und später (ab 1734) in Göttingen George Christian Gebauer in vier Bänden vorlegte. Er flankierte diese umfassende Edition des Romans mit einer ihrerseits ausufernden „Vorrede an den Leser“, in der er den Roman insbesondere dafür hochhält, „dass Herr von Lohenstein der erste einen vollkommenen Sprach-Schatz, in welchem zugleich alle Wissenschaften, sonderlich die Staats-Klugheit enthalten, an den Tag gelegt“ (Gebauer 1731, $\mathrm{XX})$. Er findet in dem Werk 
nicht nur den Reichthum der teutschen Sprach eröffnet, und was immer in der Welt in allen Wissenschaften vorkommet, mit treflichen und genugsamen Red-Arten durchgehend ausgetrucket [...]; sonder zugleich auch seine weitleuffige Gelehrsamkeit [...]; von der Wissenschaft und Beschaffenheit der natürlichen Dingen; von dem Geheimnuß der wahren StaatsKlugheit; von den merckwürdigen Sitten und Gebräuchen der alten Völkern [...]. In diesem Werk findet der Gott-Gelehrte die herrlichsten Betrachtungen von Gott und seinem Wesen. Der Staats-Gelehrte findet die vollkommene Kunst, Stätt und Länder vernünftig zu beherrschen, und durch weise Gesetz glückselig zu machen. Der Naturkündiger findet die Betrachtung der irrdischen Leibern, und so viel seltsame Würkungen der Natur, die er vergeblich an andern Orten suchen würde. Der Sittenlehrer findet ein natürliche Abschilderung der unordentlichen Gemüths-Bewegungen der Menschen, samt dem Mittel selbige zu hemmen, und hernach vergnügt und glückselig zu sein. Der Kriegsmann [...] findet von Schlachten, von Zweykampf, von Belagerung und Bestürmungen [...]; und endlich findet der Liebhaber der Jahrzeit Büchern und des Alterthums, wodurch er sein verlangen stillen kan; so daß kein Stand und Alter, dem die Lesung dieses Buchs nicht in alle weg nutzlich und angenehm seyn kann, weilen der grungelehrte Lohenstein, der eine lebendige Bibliothec gewesen, seine grossen Schatz vor seinem Ableben ausgeschüttet, und seinen Teutschen in seinem Arminius übergeben. (Gebauer 1731, XXIf.)

Es ist nur konsequent, wenn Gebauer auf dieser Grundlage die Form des Registers nicht bloß aufgreift, sondern auch ausbaut: Sein Register der vornehmsten in allen vier Theilen des Arminius und der Thusnelda befindlichen Sachen ist allgemeiner angelegt als das seines Vorgängers Wagner; es enthält zahlreiche neue Lemmata und erstreckt sich im vierten Band über 86 doppelspaltige Seiten.

Was von Thomasius bis Männling und Gebauer am Arminius als das enzyklopädische Potenzial des Romans hervorgehoben wurde, konnte allerdings unter neuen poetologischen wie epistemologischen Prämissen auch Gegenstand der Kritik werden. Insbesondere aus der Perspektive der Aufklärung mit ihren neuen Kategorien - Zweifel, Selbstdenken, Vernunft - mussten die polyhistorischen romanförmigen Zentrifugen des Wissens als Ballast von überbordender Information und unökonomischen Schreibweisen gelten, dem eine schlichtere Ästhetik und eine kritische Urteilskraft entgegengehalten wurde. Programmatisch daher das Lob des Vergessens bei Georg Friedrich Meier in den Anfangsgründen aller schönen Wissenschaften (1755): „Die Vergessungskunst ist also so nöthig und nützlich, als die Gedächtniskunst“ (Meier 1755, 445). Gegenstand der Kritik wurde dabei auch die Roman-Lexikographie im Stil Männlings, beispielhaft in Jonathan Swifts Tale of a Tub (1704). Der ironische „Exkurs zum Lobe der Exkurse“ des Tonnenmärchens persifliert exakt die polyhistorische „Kunst des Ausuferns“ und seine „Mode, fünfzig Dinge in einem Gericht zusammenzuwürfeln“ (Swift 1994, 126, vgl. Swift 1958, 143).

Was bleibt uns also übrig, als unsere letzte Zuflucht zu umfangreichen Indizes und kleinen Kompendien zu nehmen? Zitate müssen zuhauf gesammelt und alphabetisch geordnet 


\begin{abstract}
werden, zu welchem Behufe zwar nicht die Autoren, wohl aber die Kritiker, Kommentatoren und Lexika sorgfältig zu konsultieren sind. Und vor allem müssen wir uns an jene gescheiten Sammler glanzvoller Passagen, Sentenzen und Merksätze halten, die von einigen als Siebe und Schüttelroste der Gelehrsamkeit bezeichnet werden. [...] Auf diese Weise etabliert sich innerhalb weniger Wochen mancher Schriftsteller, der dann in der Lage ist, die schwierigsten und umfassendsten Themen zu behandeln. Denn was macht es schon, daß sein Kopf leer ist, wenn nur seine Zitatenkladde überquillt? (Swift 1994, 131f.)
\end{abstract}

Wer auf diese Weise die Funktion des Denkens und des Gedächtnisses außer Kraft gesetzt und damit Wissen überhaupt verunmöglicht sah, konnte sich auf Aristoteles' Konzept des organischen Ganzen berufen, das in der Poetik folgendermaßen bestimmt wird: „Demzufolge müssen, wie bei Gegenständen und Lebewesen eine bestimmte Größe erforderlich ist und diese übersichtlich sein soll, so auch die Handlungen eine bestimmte Ausdehnung haben, und zwar eine Ausdehnung, die sich dem Gedächtnis leicht einprägt“ (Aristoteles 1982, 27). Hierauf bezog sich auch der Schweizer Theologie und scharfe Arminius-Kritiker Gotthard Heidegger, wenn er in der Mythoscopia Romantica (1698) monierte:

Es sollte einer wol etlich mahl die Heil. Bibel durchlesen können / eh er mit dem grossen und schwähren Arminio zu End kommet. Er schüttet dem Leser den Kopf so voll / daß es anders alß zu Nachtheil andrer Studien nicht gedeyen kan. Seine Erzehlungen sind [...] so abscheulich verviertheilt / und an weit entlegne Ohrt zerstreuet / daß die das Gemüth ohne greuliche fatiques und abschleiffen des Gedechtnuß nicht zusamen schmiegen kan. (Heidegger 1969, 88)

Anstatt eines geordneten und memorierbaren Wissens bietet der Roman eine unüberschaubare, daher nicht-memorierbare Datenflut auf. Während Thomasius im Roman gerade die Gedächtnisfunktion des Wissens garantiert sah, war nach Heidegger im Arminius eben diese Funktion außer Kraft gesetzt: Solche Romane

nehmen den Kopff gantz als in Arrest / setzen den Menschen in ein Schwitzbad der Passionen [...] machen Melancholicos und Duckmäuser / [...] den zu andrem tüchtig gewesten Geist machen sie träg und überdrüssig / betauben und belästigen das Gedechtnuß [...] verhinderen Geschäfft und studiern / und endlich an statt Wissenschafft beyzubringen scharren sie etwas zusamen / das schlimmer ist als jede Ohnwissenheit. (Heidegger 1969, 70f.)

Die Kritik galt damit der Behauptung der Literatur als Wissensform überhaupt. Sie richtete sich weniger gegen die funktionale bzw. didaktische Pragmatisierung der Literatur, der nach wie vor ein umfassender wissenschaftlicher Bildungsauftrag zugesprochen wurde. Die Kritik richtete sich vor allem gegen die formalen Konsequenzen, die sich bei der ästhetischen Realisierung dieses Auftrags ergaben. Auf ästhetischer Ebene wurde der polyhistorische Roman mit dem pole- 
mischen Begriff des ,Schwulstes“ kritisiert, auf epistemologischer Ebene galt die Kritik den enzyklopädischen Aufschreibesystemen. Mit seinen enzyklopädischen Schreibweisen - Digression, Anmerkungen, Register, Topik - strapazierte der polyhistorische Roman sowohl die neuen Stilvorgaben als auch den neuen Begriff eines kritischen und autonom erworbenen Wissens. Mit der Domestizierung der Form veränderte sich im 18. Jahrhundert aber auch die Funktion der Literatur als Wissensspeicher. Aus den polyhistorischen Enzyklopädien in Romanform wurden bürgerliche moralische Lehrstücke, aus dem Universalwissen die psychologische Charakterbildung eines Individuums. Die bürgerlichen Romane und Dramen verstreuen und registrieren nicht mehr die Daten einer ganzen Bibliothek, sondern exemplifizieren einen moralischen Lehrsatz und entwickeln, was verwickelt in einem Subjekt angelegt ist, in dramatischer und narrativer Linearität.

Diese Abwehr des barocken polyhistorischen Romans hallt noch in der Literaturgeschichtsschreibung des 19. Jahrhunderts wider - markant in Joseph von Eichendorffs Geschichte der poetischen Literatur Deutschlands (1857). Dem Barockroman gehe es, so Eichendorff, „lediglich um eine breite Schaustellung ihrer Gelehrsamkeit“, indem ,alle erdenklichen Artikel des Wissens [...] mit großer Präsentation und Selbstschätzung an die lernbegierige Lesewelt ausgeboten werden. Man könnte ihre Romane poetische, gewissermaßen tollgewordene Realencyklopädien nennen“ (Eichendorff 1970, 111). „Kein Wunder daher“, so fährt er fort, „daß fast jeder dieser Romane mehrere Foliobände füllt, wie denn z.B. die ,Aramena‘ der Herzogs Ulrich von Braunschweig nicht weniger als 6822 Seiten enthält“ (Eichendorff 1970, 109). Als extremes Beispiel nennt auch er Lohensteins Arminius, der alle Romane dieses Typus „überbot“, indem er „endlich Alles, was damals die Gelehrten wußten oder zu wissen sich einbildeten, Geographie, Völker- und Länderkunde, Astrologie und Geschichte unausreißlich fest in Einen ungeheueren Zopf zusammengebunden“ (Eichendorff 1970, 112).

Die Verknüpfung literarischer und wissenschaftlicher Gattungen und Ansprüche erweist sich auch für Eichendorff als Aufkündigung des klassischen Postulats des „organischen Ganzen“, mit der Konsequenz der Zerstörung sowohl von Kunst als auch von Wissen: „Was uns [...] fast schreckhaft an ihnen [= den Romanen des Barock] auffällt, ist ihre monströse Unförmlichkeit, eine elementarische Confusion aller möglichen und unmöglichen Dichtungsarten, die hier chaotisch nebeneinander liegen und kaum noch den Versuch machen, sich zu einem organischen Ganzen zu gestalten“ (Eichendorff 1970, 109). Nach dieser Lesart mündet der polyhistorische Roman des Barock gerade durch seine Hybridisierung von Literatur und Wissen in einer Deformation, ja Destruktion sowohl von Wissen als auch von Literatur. 


\section{Literatur}

Aristoteles. Poetik. Griechisch/Deutsch. Übers. und hg. von Manfred Fuhrmann. Stuttgart: Reclam, 1982.

Aulus Gellius. Die Attischen Nächte. Übers. von Fritz Weiss. Leipzig: Fues, 1875.

Borgstedt, Thomas. Reichsidee und Liebesethik. Eine Rekonstruktion des Lohensteinischen Arminiusromans. Tübingen: Niemeyer, 1992.

Budé, Guillaume. De philologia, in: Opera omnia, Basel 1557. Zitiert nach: Kühlmann, Wilhelm, Gelehrtenrepublik und Fürstenstaat, Entwicklung und Kritik des deutschen Späthumanismus in der Literatur des Barockzeitalters, Tübingen: Niemeyer, 1982. 289.

Eichendorff, Joseph von. Geschichte der poetischen Literatur Deutschlands (1857). Sämtliche Werke. Historisch-kritische Ausgabe. Hg. von Wolfgang Mauser. Bd. 9. Regensburg: Habbel, 1970.

Garzoni, Tommaso. Piazza universale, Das ist: Allgemeiner Schawplatz / oder Marckt / vnd Zusammenkunfft aller Professionen / Künsten / Geschäfften / Händeln vnd Handtwercken / so in der gantzen Welt geübet werden (1619). Frankfurt a. M.: Jennisius, 1626.

Gebauer, George Christian. „Vorrede an den Leser.“ Daniel Casper von Lohenstein: Großmüthiger Feld-Herr Arminius oder Herrmann. Bd. 1. Leipzig: Gleditsch, 1731. I-LVI.

Grimmelshausen, Hans Jakob Christoffel von. Der abentheuerliche Simplicissimus teutsch und Continuatio des abentheuerlichen Simplicissimi. Hg. von Rolf Tarot. Tübingen: Niemeyer, 1967.

Harsdörffer, Georg Philipp. Deliciae Physico Mathematicae. Oder Mathemat. und Philosophische Erquickstunden, Dritter Theil, Bestehend in Fünffhundert nutzlichen und lustigen Kunstfragen/ und deroselben gründlichen Erklärung: Mit vielen nothwendigen Figuren/ so wol in Kupffer als Holtz/ gezieret. Nürnberg: Endter, 1653.

- Frauenzimmer Gesprächsspiele (1641-1649). Bd. 4. Tübingen: Niemeyer, 1968/1969.

Härter, Andreas. Digression. Studien zum Verhältnis von Ordnung und Abschweifung in Rhetorik und Poetik. Quintilian - Opitz - Gottsched - Friedrich Schlegel. München: Fink, 2000.

Heidegger, Gotthard. Mythoscopia Romantica oder Discours von den so benanten Romans. Faksimileausgabe nach dem Originaldruck von 1698. Bad Homburg: Gehlen, 1969.

Heselhaus, Clemens. Anton Ulrichs Aramena. Studien zur dichterischen Struktur des deutschbarocken ,Geschichtgedicht'. Würzburg: Triltsch, 1983.

Hofmann, Caspar. De barbarie imminente (1578), Nürnberg 1726. Zitiert nach: Kühlmann, Wilhelm. „Lektüre für Bürger. Eigenart und Vermittlungsfunktion der polyhistorischen Reihenwerke Martin Zeillers (1589-1661).“ Literatur und Volk im 17. Jahrhundert. Probleme populärer Kultur in Deutschland. Hg. von Wolfgang Brückner, Peter Bickle, Dieter Breuer. Wiesbaden: Harrassowitz, 1985. 917-934.

Huet, Pierre Daniel. „Traité de l’origine des romans.“ in: Theorie und Technik des Romans im 17. und 18. Jahrhundert. Hg. von Dieter Kimpel, Conrad Wiedemann. Bd. 1. Tübingen: Niemeyer, 1970.

Kafitz, Dieter. Lohensteins ,Arminius‘. Disputatorisches Verfahren und Lehrgehalt in einem Roman zwischen Barock und Aufklärung. Stuttgart: Metzler, 1970.

Kilcher, Andreas. Mathesis und Poiesis. Die Enzyklopädik der Literatur 1600-2000. München: Fink, 2003.

- „Theorie des alphabetisierten Textes.“ Allgemeinwissen und Gesellschaft. Hg. von Paul Michel. Aachen: Shaker, 2007. 75-94. 
Kraft, Stephan. Geschlossenheit und Offenheit der, Römischen Octavia'von Herzog Anton Ulrich. Würzburg: Königshausen und Neumann, 2004.

Kuhlmann, Quirinus. Himmlische Libes-küsse (1671). Tübingen: Niemeyer, 1971.

- Lehrreicher Geschicht-Herold oder Freudige und traurige Begebenheiten Hoher und Nidriger Personen: Welche Theils nach der neu-vermehrten Wunderart des weltberuffenen Athanasius Kircher vorgetragen [...]. Jena: Meuer, 1673.

Kuhlmann, Quirinus. Neubegeisterter Böhme (1674). Neu hg. und erläutert von Jonathan Clark. Stuttgart: Hiersemann, 1995.

Link-Heer, Ursula. „Zur Kanonisierung antiklassischer Stile. Manierismus und Barock“. Kanon, Macht, Kultur. Theoretische, historische und soziale Aspekte ästhetischer Kanonbildungen. Hg. von Renate von Heydebrand. Stuttgart: Metzler, 1998. 156-176.

Lohenstein, Daniel Casper von. Großmüthiger Feldherr Arminius (1689-90). 2 Bde. Bern: Lang, 1973.

Lullus, Raimundus. Ars brevis. Lateinisch - Deutsch. Übers., mit einer Einleitung hg. von Alexander Fidora. Hamburg: Meiner, 1999.

Männling, Johann Christoph. Arminius enucleatus. Das ist: Des unvergleichlichen Daniel Caspari von Lohenstein/ Herrliche realia, Köstliche Similia, Vortreffliche Historien/ Merkwürdige Sententien, und sonderbare Reden. Als Köstliche Perlen und Edelgesteine aus dessen deutschen Tractati oder Arminii. 2 Bde. Leipzig: Ernsten \& Jenisch, 1708.

Martino, Alberto. Daniel Caspar von Lohenstein. Geschichte seiner Rezeption. Bd.1: 1661-1800. Tübingen: Niemeyer, 1978.

Meier, Georg Friedrich. Anfangsgründe aller schönen Wissenschaften. Zweyter Theil, andere Auflage. Halle: Hemmerde, 1755.

Moser, Walter. Romantisme et crises de la modernité. Poésie et encyclopédie dans le „Brouillon“ de Novalis. Longueuil, Québec: Préambule, 1989.

Müller-Bochat, Eberhard. „Die Einheit des Wissens und das Epos. Zur Geschichte eines utopischen Gattungsbegriffs.“ Romanistisches Jahrbuch 17 (1966): 58-81.

Neubauer, John. Symbolismus und symbolische Logik. Die Idee der Ars Combinatoria in der Entwicklung der modernen Dichtung. München: Fink, 1978.

Nikolaus von Kues. Die belehrte Unwissenheit. De docta ignorantia. Hg. von Hans Gerhard Senger. Hamburg: Meiner, 1977.

Rieger, Stefan. Speichern/Merken. Die künstlichen Intelligenzen des Barock. München: Fink, 1997. Rossi, Paolo. Clavis universalis. Arti mnemotechnice e logica combinatoria da Lullo a Leibniz. Milano: Riccardi 1960.

- „The legacy of Ramon Lull in sixteenth-century thought.“ Medieval and Renaissance Studies 5 (1961): 182-213.

- „Universal Languages, Classifications and Nomenclatures in the Seventeenth Century. “ History and Philosophy of the Life Sciences 6 (1984): 119-131.

Schmidt-Biggemann, Wilhelm. Topica universalis. Eine Modellgeschichte humanistischer und barocker Wissenschaft. Hamburg: Meiner, 1983.

Schock, Flemming. Die Text-Kunstkammer. Populäre Wissenssammlungen des Barock am Beispiel der ,Relationes Curiosae‘von E.W. Happel. Köln, Weimar, Wien: Böhlau, 2011.

Schock, Flemming, Hg., Polyhistorismus und Buntschriftstellerei. Populäre Wissensformen und Wissenskultur in der Frühen Neuzeit. Berlin, New York: De Gruyter, 2012.

Siegel, Steffen. „Architektur des Wissens. Die figurative Ordnung der artes in Gregor Reischs Margarita Philosophica.“ Das Bild als Autorität. Die normierende Kraft des Bildes. Hg. von Frank Büttner, Gabriele Wimböck. Münster: LIT Verlag, 2004. 343-362. 
Swift, Jonathan. A Tale of a Tub. To which is Added the Battle of the Book and the Mechanical Operation of the Spirit. Hg. von Adolf Chales Guthkelch, D. Nichol Smith. Oxford: Claredon, 1958.

- Ein Tonnenmärchen. Übers. von Ulrich Horstmann. Stuttgart: Reclam, 1994.

Szarota, Elida Maria. Lohensteins Arminius als Zeitroman. Sichtweisen des Spätbarock. München: Francke, 1970.

Texte zur Romantheorie I (1626-1732). Mit Anmerkungen, Nachwort und Bibliographie hg. von Ernst Weber. München: Fink, 1974.

Thomasius, Christian. „Monats-Gespräche.“ Theorie und Technik des Romans im 17. und 18. Jahrhundert. Hg. von Dieter Kimpel, Conrad Wiedemann, Bd. 1. Tübingen: Niemeyer, 1970.

Vosskamp, Wilhelm. Romantheorie in Deutschland. Von Martin Opitz bis Friedrich von Blanckenburg. Stuttgart: Metzler, 1973.

Wagner, Christian. Anmerckungen über Herrn Daniel Caspers von Lohenstein Arminius. Nebst beygefügtem Register derer in selbigem Werck befindlichen merckwürdigen Nahmen und Sachen. Leipzig: Gleditsch, 1690.

Welzig, Werner. „Einige Aspekte barocker Romanregister.“ Stadt - Schule - Universität - Buchwesen und die deutsche Literatur im 17. Jh. Hg. von Albrecht Schöne. München: Beck, 1976. 562-570.

Yates, Frances A. Giordano Bruno and the Hermetic Tradition. London: Routledge and Kegan Paul, 1964.

Zeller, Rosmarie. Spiel und Konversation im Barock. Untersuchungen zu Harsdörffers ,Gesprächsspielen‘. Berlin, New York: de Gruyter, 1974. 